\title{
Why Five Fingers? \\ Evolutionary Constraints on Digit Numbers
}

Frietson Galis (galis@rulsfb.leidenuniv.nl) Jacques J.M. van Alphen (alphen@rulsfb,leidenuniv.nl) Johan A.J. Metz (metz@rulsfb.leidenuniv.nl)

\section{Approved by}

Ulf Dieckmann

Project Leader, Adaptive Dynamics Network

May 2001 Institute, its National Member Organizations, or other organizations supporting the work. 


\section{Contents}

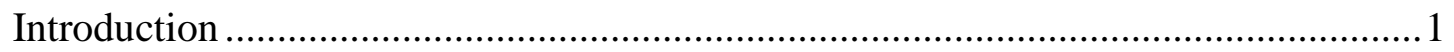

Selective advantage of changes in digit number ...................................................... 3

Polydactyly, modularity and the phylotypic stage …............................................. 4

Molecular pathways of limb and digit development ...............................................

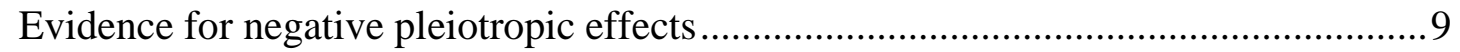

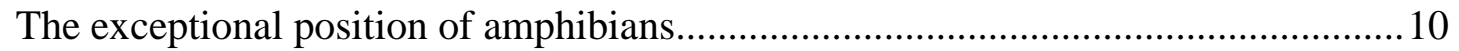

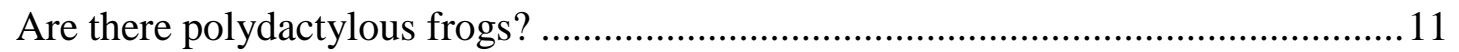

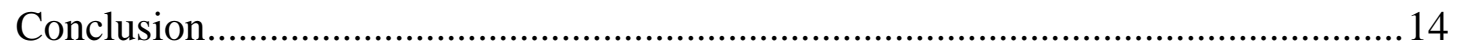

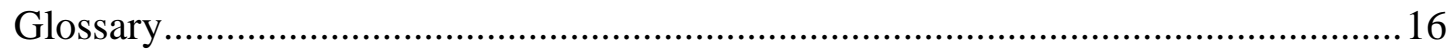

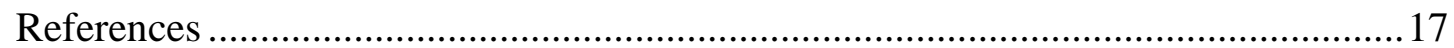




\begin{abstract}
Evolutionary changes in the number of digits and other limb elements appear to be severely constrained, probably as a result of a low level of modularity during limb development. Reduced limb structures typically develop through a process of construction followed by destruction and amniotes have evolved many digit-like structures rather than actual extra digits. In amniotes, limb development occurs during the crucial phylotypic stage, when many inductive interactions are occurring throughout the body. As a result, changes in limb development usually engender changes in other body parts. Thus, mutations that change the number of limb bones are expected to have many pleiotropic effects, which severely reduces the chance of such mutations being successful. In amphibians with aquatic larvae, limb development occurs after the phylotypic stage and limb development is decoupled from the interactivity of the phylotypic stage. The constraint of pleiotropic effects is, therefore, expected to be weaker. This expectation agrees with the larger variability in the number of hand and foot structures in amphibians, with frogs even occasionally possessing six toes. These facts once again emphasize the importance of pleiotropic effects as constraints to evolutionary change, including their role in the conservation of body plans.
\end{abstract}




\title{
About the Authors
}

\author{
Frietson Galis \\ Jacques J.M. van Alphen \\ Institute of Evolutionary and Ecological Sciences \\ Leiden University \\ Kaiserstraat 63, 2311 GP Leiden, The Netherlands \\ Johan A.J. Metz \\ Institute of Evolutionary and Ecological Sciences \\ Leiden University \\ Kaiserstraat 63, 2311 GP Leiden, The Netherlands \\ and \\ Adaptive Dynamics Network \\ International Institute for Applied Systems Analysis \\ Schlossplatz 1, 2361 Laxenburg, Austria
}

\section{Acknowledgments}

We dedicate this article to Karel Liem for his perceptive insights and his kindness. We thank Jeff Levinton for alerting us to the polydactylous monsters of Sewall Wright; Günter Wagner and Russ Lande for stimulating and helpful discussions; Marc Nussbaum for information on Berner Mountain dogs; Marty Cohn for information on the digital arch model; Martin Brittijn Adri't Hooft, Peter van Mulken and for help with the figures; and Brian Hall, Russ Lande, Armand LeRoi and Günter Wagner for insightful comments. 


\title{
Why Five Fingers? \\ Evolutionary Constraints on Digit Numbers
}

\author{
Frietson Galis \\ Jacques J.M. van Alphen \\ Johan A.J. Metz
}

\section{Introduction}

Reductions in the number of digits have occurred frequently during tetrapod evolution. Further reductions in the number of limb bones have also occurred, including the complete elimination of limbs in, for example, snakes and caecilians. Strikingly, an evolutionary reversal of limb reduction has never occurred ${ }^{1,2}$. This is puzzling when one considers that mutations for POLYDACTYLY (see Glossary) are particularly common. In humans, for example, the most common anomaly at birth is the presence of an extra toe or finger ${ }^{3,4}(0.1-0.2 \%)$. The extra digit can be rudimentary, or fully formed and functional. Polydactyly, either as an evolutionary novelty, or as a result of ATAVISM, is a common phenomenon in other tetrapods ${ }^{5}$ (Fig. 1). The horses of Alexander the Great and Julius Caesar, for example, were reported to have three toes and to be of legendary excellence. The absence of polydactyly in evolution is clearly not an absence of heritable variation for the trait. Therefore, there must be selection against mutations causing polydactyly or other reversals of limb reduction.

Lande $^{1}$ and Wright $^{2}$ have hypothesized that polydactyly is not selected against directly. They argue that selection against mutations causing polydactyly occurs because of negative PLEIOTROPIC effects. Lande ${ }^{1}$ argues that any change in the number of limb bones is usually selected against. For decreases there is, however, an evolutionary way around the constraint: construction followed by destruction. In the initial evolutionary steps of limb reduction, the original number of digits appears as ANLAGE (Fig. 2a). The development of the Anlage of one digit, usually the last one formed, is then followed by regression in its size, resulting in only a rudimentary digit (Fig. 2b; Box 1). This can still be observed in most species with a reduced number of digits, such as the Anlage of the postminimus (a sixth finger) in humans ${ }^{6}$. When limb reduction is continued during evolution, the development of Anlagen of both bone and muscle, is slowly reduced. As far as we know, continued limb reduction always follows the process of construction and destruction. In reptiles, the regression of Anlagen is the result of cell death ${ }^{1,7}$ once the limb has started to develop. For example, in the limbless lizard Ophisaurus apodus, the limb buds develop, and myotomes that normally develop into limb muscles extend from the somite into the limb bud, where they distribute cells. Necrosis then begins, first in the ridge, followed by the myotomal cells and then the mesenchymal cells. At first, the limb bud continues to grow during the necrosis, but it then regresses and forms only tiny rudiments of most of the limb bones ${ }^{8}$. 


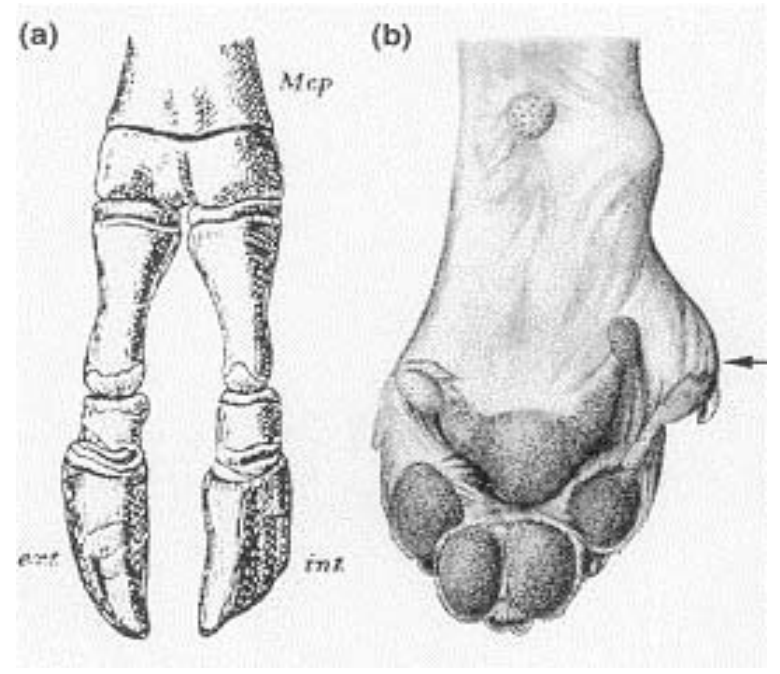

Fig. 1. Examples of polydactyly. (a) Foot of horse with two toes. Reproduced from Ref. 5. (b) Foot of St Bernard dog with five toes, including an atavistic first digit (arrow). In wolves and most other canids, the first toe is reduced, and they only have four toes. Reproduced from Ref. 61.
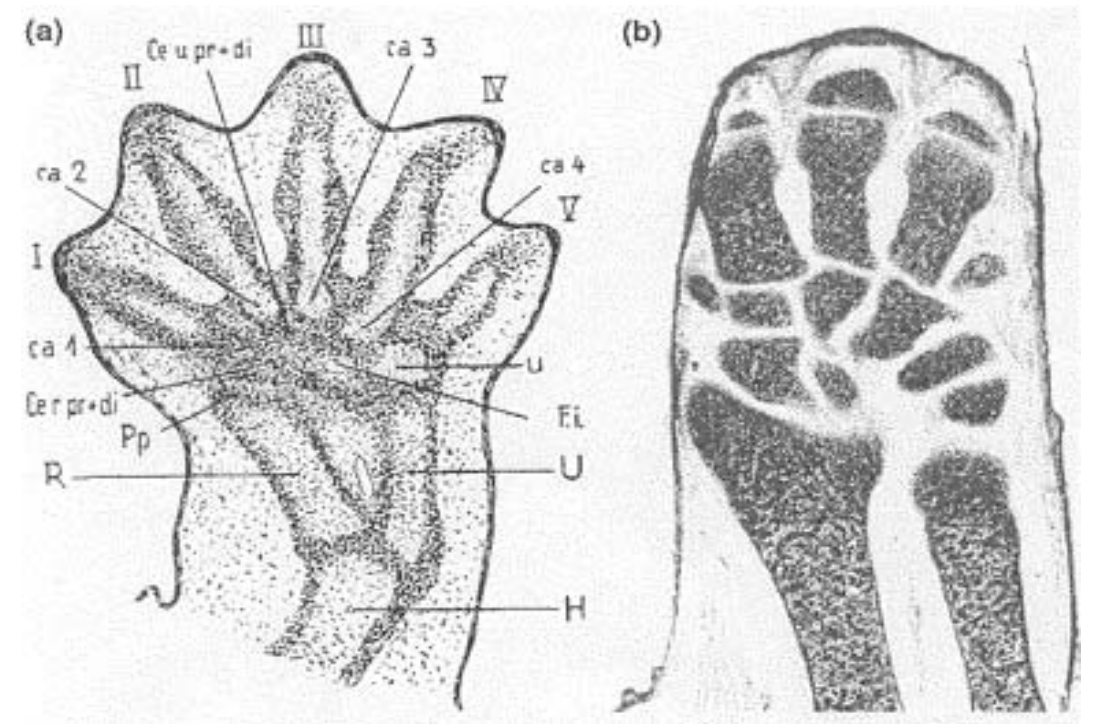

Fig. 2. Evolutionary reduction by construction and destruction. (a) Embryonic hand of the opossum Didelphis marsupialis, showing full-sized Anlagen of all five digits. Abbreviations: H, humerus; R, radius; pp, prepollex; $\mathrm{U}$, ulna; u, ulnare; I-V, digits). (b) hand of juvenile of D. marsupialis, showing a reduced first and fifth digit. Reproduced, with permission, from Ref. 6.

This mechanism of evolutionary limb reduction suggests that the construction phase cannot easily be missed during normal development. The most probable reason for this is that the inductive interactions of the construction phase of the limb cannot be missed out of the chain of inductive interactions of opera development. This conclusion is in agreement with the extremely slow speed of evolutionary limb reduction. 


\section{Box 1. Polydactyly in dogs}

In general, large dogs frequently have extra rudimentary or fully formed toes, a high rate of locomotory diseases and high mortality rates ${ }^{b}$ (often not living beyond six years of age, whereas large mammals generally die at a higher age than small mammals). In St Bernard dogs and Bernese mountain dogs, the first toe, commonly reduced in canids, is usually present in the hindlimb (Fig. 1b) and is often double in St Bernards because of a history of artificial selection for this character $^{c-e}$. The death rate of St Bernards and Bernese mountain dogs is very high $^{\mathrm{b}}$. Locomotory diseases (elbow and hip joint problems), as well as tumors and heart disease, are a common cause of death in these dogs. The rare and small Norwegian Lundehunds, which are used for hunting puffins, are required to have a minimum of six toes on their hindlimbs, according to the breed standard. The extra toes presumably prevent them from slipping off the rocks. These dogs have extremely high rates of gastritis and intestinal Iymphangiecstasia. In addition, $30 \%$ of 14 autopsied dogs in a 13-year study suffered from gastric carcinoma ${ }^{f}$. The serious health problems in dogs with polydactyly are in agreement with the assumption that the pleiotropic effects of mutations tor polydactyly are likely to be serious in other parts of the limb and in other parts of the body. Further research is necessary to test which of these problems in dogs are, indeed, linked to polydactyly and which are independent by-products of inbreeding.

\section{References}

a Alberch, P. (1985) Developmental constraints: why St. Bernards often have an extra digit and poodles never do. Am. Nat. 126:430-433

b Bonnett, B.N. et al. (1997) Mortality in insured Swedish dogs: rates and causes of death in various breeds. Vet. Rec. 141:40-44

c Lande, R. (1978) Evolutionary mechanisms of limb loss in tetrapods. Evolution 32:73-92

d Seiferle, E. (1927) Atavismus und Polydaktylie der hyperdaktilen Hinterpfote des Haushundes. Morph. Jb. 57:313-380

e Willis, M.B. (2000) Dew claw removal in Bemese mountain dogs. Vet. Rec. 147:84

f Kolbjornsen, O. et al. (1994) Gastropathiesin the Lundehund. II. A study of mucin profiles. Acta Pathol. Microbiol. Immunol. Scand. 102:801-809

\section{Selective advantage of changes in digit number}

Under certain circumstances, there are selective advantages to having fewer toes (e.g. for running ${ }^{9}$ ), or even completely reduced legs. The reduction in the number of toes of ostriches parallels that seen in ungulates. Similarly, in burrowing organisms from different vertebrate groups, the limbs have become reduced independently many times in association with body elongation (e.g. in snakes), or hands with special digging devices have evolved, as in moles Talpa europaea.

Extra digits on the hand give further support in swimming and digging for some animals, and a better grasp of branches in tree-dwelling species. In amniotes, extra digitlike structures have evolved in association with these functions many times (Fig. 3). However, these extra structures are never 'true' digits but are, for example, modified 
wrist bones or extra phalanges. Ichthyosaurs were long thought to have evolved true polydactyly, possessing up to nine digits. However, recent insights suggest that they never had more than five digits and that there were many SUPERNUMERARY PHALANGES that spread out over many rays, possibly by bifurcations of those rays ${ }^{10,11}$. In moles, there is an impressive digit-like structure that functions in digging (Fig. 3a). Pandas Aeluropus spp. even have two extra digit-like structures: the well-known thumb that is not a 'true' digit, and an extra digit-like structure on the side of the little finger ${ }^{12}$. The 'thumb' is a sesamoid bone and the extra little finger an enlarged wrist bone, the pisiform. In mosasaurs, whales and turtles, the paddle is strengthened by an extra digitlike structure, which, as in pandas, is an enlarged pisiform (Fig. 3b). Another evolutionary solution for extra digits for swimming or grasping is a change of the backward-pointing toe into a forward-pointing toe, or vice versa (Box 2).

The repeated selection for extra digit-like structures across a range of animals shows that extra digits can have a strong functional advantage; however, this does not translate into a selective advantage for polydactyly. As already observed by Lande ${ }^{1}$ and $\mathrm{Wright}^{2}$, the constraint on polydactyly cannot be explained by negative selection against the extra digits themselves. There must, therefore, be strong negative pleiotropic effects associated with polydactyly that indirectly pose an evolutionary constraint.

The recent increase in molecular biology data and the emergence of evolutionary developmental biology provide new means with which to study the pleiotropic effects associated with polydactyly.

\section{Polydactyly, modularity and the phylotypic stage}

In amniotes, the period of limb patterning occurs mainly in the phylotypic stage, which is the stage at which vertebrates most resemble each other ${ }^{13}$. This stage begins with neurulation and ends when the majority of somites have been formed. We propose that the constraint on changes in the number of digits is part of the constraint on changes in this stage.

\section{Pleiotropy and the phylotypic stage}

The phylotypic stage is characterized by intense INDUCTIVE SIGNALING between different parts of the embryo, including somites, notochord, neural tube, latera1 plate mesoderm and limb buds. The presence of inductive interactions between different body parts causes mutational changes in one part of the body to have pleiotropic effects in other parts of the body. Raff ${ }^{14}$, refining a hypothesis of Sander ${ }^{15}$, hypothesized that this widespread global INDUCTIVE INTERACTIVITY is the cause of the conservation of the phylotypic stage. The pleiotropic effects that Lande ${ }^{1}$ and Wright $^{2}$ proposed as the cause of the conservation of the number of limb bones could, thus, be part of the pleiotropic effects that appear to constrain mutational changes during this stage in general. Lande's ${ }^{1}$ and Wright's ${ }^{2}$ hypotheses would then become more specific: the evolutionary constraint on polydactyly and other reversals of limb reduction is caused by the high interactivity among body parts (MODULES) during the phylotypic stage.

\section{Global inductive interactions constrain mutational changes}

Galis and Metz ${ }^{16}$ recently tested the hypotheses of Sander ${ }^{14}$ and Raff ${ }^{15}$ by analyzing teratological studies that reported effects of phenocopies of mutational changes (i.e. induced phenotypic changes that mimic mutations). The analysis provides strong support for the assumption that the many global inductive interactions during the 


\section{Box 2. Homeotic changes of digit identity}

In aquatic birds, an increase in paddle stiffness is not obtained via polydactyly. In many such birds, the paddle consists of three forward-pointing digits and one backward-pointing toe. This is the ancestral position of toes in birds. In the pelican family (pelicans, boobies and cormorants), the one backward-pointing toe has moved forward and is incorporated in the paddle (Fig. Ia). The absence of a backward-pointing prop for the foot could partly explain the poor walking ability of these birds, indicating a tradeoff between swimming and walking. In treedwelling birds and mammals, there is an advantage in having an extra opposed digit for grasping branches firmly. In all cases, the extra opposed digit has been obtained by changing the form of the second digit into that of the first, opposable, digit. For example, parrots, cuckoos and woodpeckers have two forward-pointing toes and two backward-pointing toes (Fig. Ib). In mammals, the ancestral number of toes is five, and so koalas have three forward-pointing toes and two backwardpointing ones. The extra backward-pointing toe is never the result of an extra digit, whereas a mutation for an extra thumb occurs, at least in humans, with a frequency of one in 5000 (Ref. a).

\section{References}

a Castilla, E.E. et al. (1996) Epidemiological analysis of rare polydactylies. Am. J. Med. Genet. 65:295-303

b Evans, T.H. (1900) The Garnbridge Natural History. Vol. 3. Birds, Macmillan

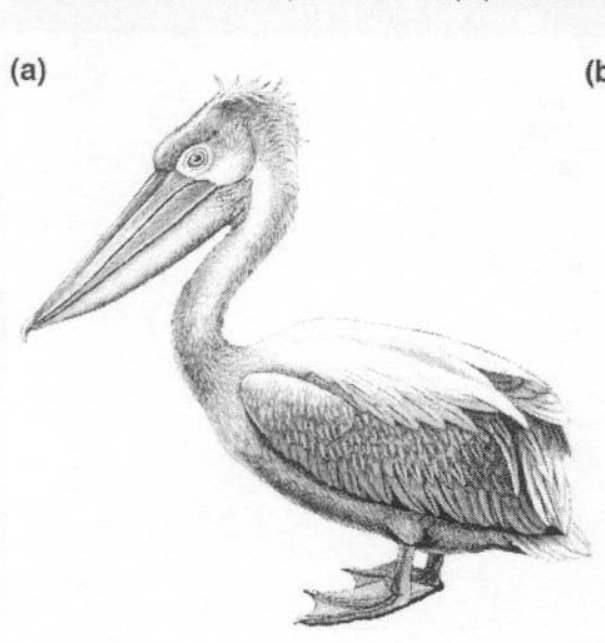

(b)

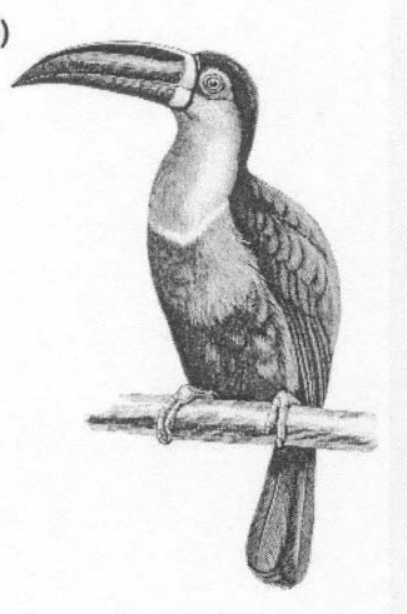

Fig. I. Position shifts of toes in birds. (a) Pelican, with four toes included in the paddle. The fourth toe has moved forward. (b) Toucan, with two forward-pointing toes and two backwardpointing ones. Reproduced from Ref. $b$.

phylotypic stage lead to negative pleiotropic effects of mutational changes acting during that stage. These effects are expected to constrain evolutionary change. This is in agreement with current ideas on the importance of MODULARITY in evolutionary change ${ }^{17-19}$. 

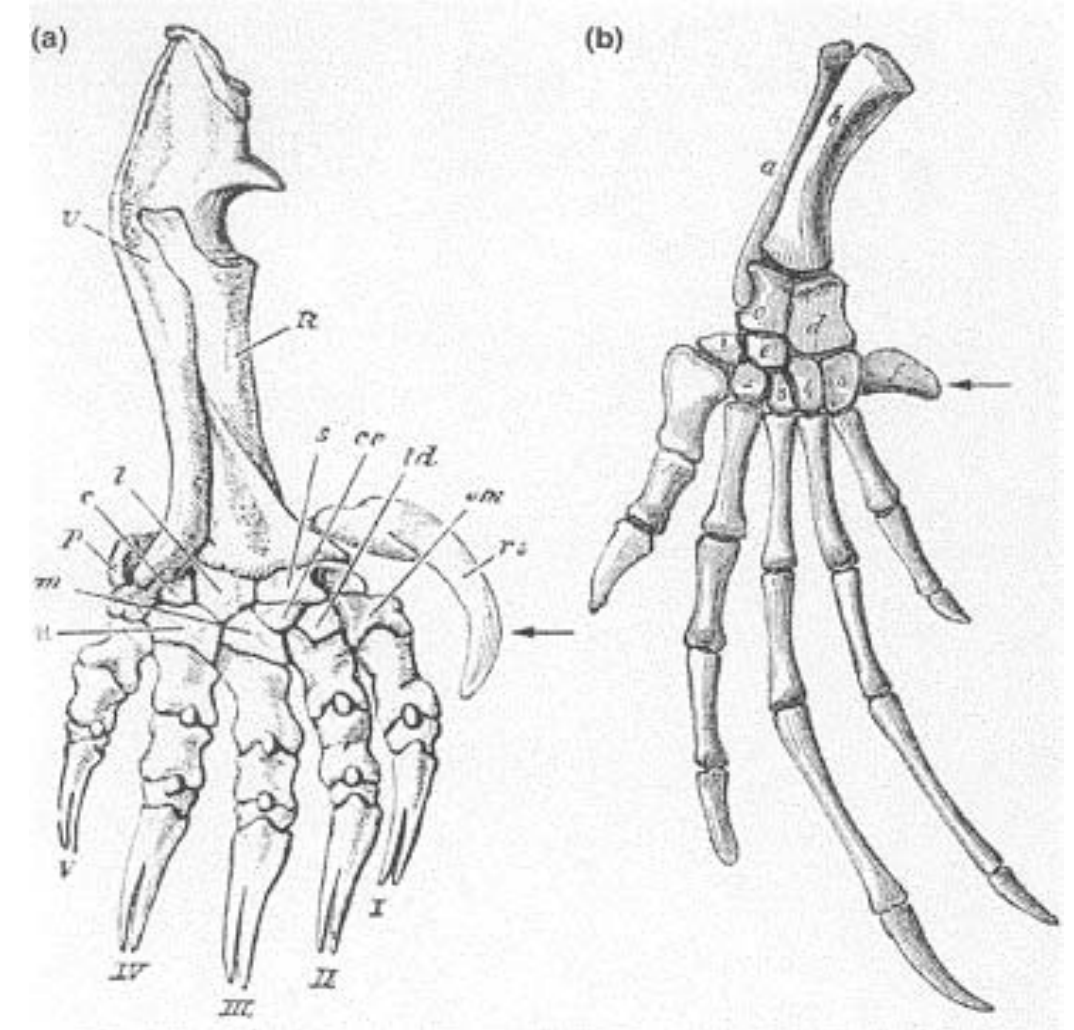

Fig. 3. Extra digitlike structures in amniotes. (a) Mole Talpa europea with extra digit-like structure (arrow). This structure is a sesamoid bone. Reproduced from Ref. 62. (b) The extra digit-like structure that contributes to the paddle of the sea turtle Chelone is an enlarged pisiform (arrow). A similar solution has evolved in whales and mosasaurs. Reproduced from Ref. 63.

\section{The timing of limb development relative to inductive interactivity}

Do these results on the strong coupling between modules during the phylotypic stage help us to understand the evolutionary constraint on polydactyly? To answer this, we need to know whether digit development of tetrapods takes place during the time when there is intense interactivity among different parts of the embryo. In mice, the period of high vulnerability resulting from global inductive interactions is from embryonic day (E) 7 to 11, and vulnerability sharply decreases thereafter (Fig. 4). Therefore, in mice, the phylotypic stage can be specified as being from E7 to E11. In these animals, the development of the limb bud starts on E8, and mesenchymal digit condensations appear on E10 and E11, in the forelimb slightly before the hind $\operatorname{limb}^{20}$. The patterning of the limb up to the determination of the number of digits therefore occurs during a stage that is characterized by global inductive interactivity. Hall and Myake ${ }^{21}$ emphasize that most of the mutations affecting skeletal development act on the early condensation stage ${ }^{21,22}$. The staging tables of Keibel $^{23}$ show that limb development occurs at roughly comparable stages in lapwings, sand lizards, deer, mice, humans and chickens. Therefore, in amniotes, unlike in the majority of amphibians, most of the limb patterning occurs during the phylotypic stage. However, limb development might not form part of the general interactivity of the phylotypic stage: it could instead form a semi-independent module. 


\section{Box 3. The developmental constraint that determines the order of limb reduction}

The digit reduced first during evolution is usually the one formed last $\mathrm{t}^{\mathrm{a}-\mathrm{c}}$.We propose the following explanation for this phenomenon: modification of the last digit formed during development will be subject to the least interference by inductive interactions. Most of the limb has already been formed and, in amniotes, the extent of its interactions with the rest of the embryo has also seriously declined. In addition, the regression in size of the limb bud by apoptosis or necrosis will be easiest with the smallest digit being formed, which is usually the last one. The mechanism proposed by Alberch and Gale ${ }^{c}$ for the evolutionary constraint of this process is not supported by evidence from processes in evolutionary limb reduction. They hypothesize that a smaller size of the mesenchymal condensation leads evolutionarily to the disappearance of the lastformed digit. This is in contrast with the described evidence that evolutionary limb reduction proceeds by a process of construction followed by destruction (Fig. 2 for mammals; Fig. 5 for amphibians).

\section{References}

a Lande, R. (1978) Evolutionary mechanisms of limb loss in tetrapods. Evolution 32:73-92

b Alberch, P. and Gale, E.A. (1983) Size dependence during the development of the amphibian foot. Cochicine-induced digital loss and reduction. J. Embryol. Exp. Morphol. 76:177-197

c Alberch, P. and Gale, E.A. (1985) A developmental analysis of an evolutionary trend: digital reduction in amphibians. Evolution 39:8-23

Is limb development in amniotes integrated in the general interactivity of the phylotypic stage?

The self-organizing capacity of the limb is very limited in amniotes. The success of transplanting limb buds in amniotes is low, in contrast to amphibians, where even transplantations to the head can be successfu $1^{24}$. Explanation experiments in mice show that, up to E12, limb buds do not develop successfully unless somitic material is also explanted. On E12 (when the vulnerability to inductive interactions has sharply decreased) the self- differentiating capacity increases markedly, and the results are considerably better on E13 and E14 (Ref. 25). Similar results were obtained for chickens $^{26}$. Therefore, the transplantation and explantation results show that the developing limb is not an independent module.

\section{Molecular pathways of limb and digit development}

The recent progress in identifying molecular pathways involved in limb patterning has revealed many dependent inductive interactions. For example, there is a complex interplay between signaling centers in the limb, the zone of polarizing activity (ZPA), the apico-epidermal ridge (AER) and the progress zone ${ }^{27,28}$. In addition, genes elsewhere play important roles ${ }^{29,30}$. If the constraint on polydactyly is due to strong coupling between modules during the phylotypic stage, it is particularly important to 


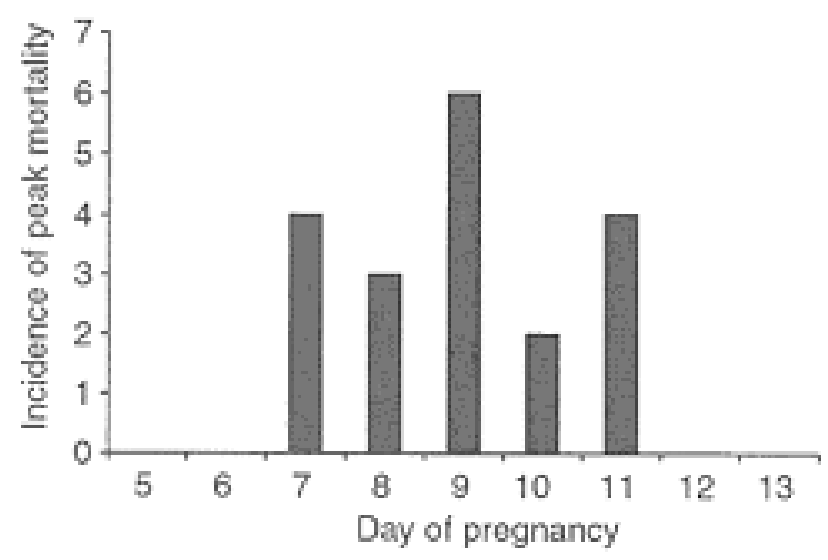

Fig. 4. The vulnerability of the phylotypic stage to induced changes (phenocopies of mutations). Vulnerability to teratogenic treatments in rodents is highest during the phylotypic stage [embryonic day (E) 7-11 in mice]. This vulnerability is caused by dependent inductive interactions. Peak sensitivity to the induction of mortality occurs on a particular day during pregnancy, always within the phylotypic stage, usually on E9. Reproduced, with permission, from Ref. 16.

know which genes are involved in patterning the digits and, in particular, which are the most upstream genes that have to be affected for polydactyly to occur. The more upstream these genes are, the more likely it will be that changing their activity also affects other parts of the embryo.

Many genes are involved in digit development and specification, including members of the Hedgehog, BmP, Fgf and Hox families, which are all relatively upstream genes. These genes, or at least a combination of them, are always involved in the development of polydactyly and oligodactyly in mice, chickens and Xenopus ${ }^{31,32}$. A crucial role seems to be played by the Sonic hedgehog gene $(S h h)$. Ectopic expression of $S h h$ in the anterior part of a developing limb bud is nearly always involved in polydactyly, and when this is not the case, ectopic anterior expression of the related Indian hedgehog gene $(I h h)$ is suspected ${ }^{30,32}$. In all cases, genes directly downstream from Shh are expressed ectopically. Recent data ${ }^{32}$ emphasize the importance of $S h h$ in the determination of both digit number and identity. Drossopoulou et al. ${ }^{32}$ suggest that $S h h$ probably acts first over a long range to control digit number and then later over a short range to induce expression of Bonemorphogeneticprotein genes $(\mathrm{BmP})$, the morphogenetic action of which specifies digit identity.

\section{Expected pleiotropic effects of polydactyly for other parts of limb patterning}

Members of the Hedgehog, BmP, Fgf and Hox families play important roles in proximo-distal, dorso-ventral and antero-posterior patterning. Multiple molecular interactions link the development along these three axes to generate the integrated system of limb development ${ }^{28}$. For example, the signaling of Shh in the ZPA that is involved in the specification of digit number (part of the antero-posterior patterning of the limb) is also involved in the proximo-distal outgrowth of the limb ${ }^{28}$. The same signals might be involved in patterning bones, tendons and muscles, because the grafting of an extra Shh expressing ZPA in the limb bud causes the development of an extra set of all these elements. This fits with the observation that Shh is involved in keeping muscle precursor cells in a proliferative state, thereby increasing the size of the muscle masses of the limb ${ }^{33}$. 


\section{Expected pleiotropic effects of polydactyly for other parts of the embryo}

It is likely that the upstream genes mentioned above are also involved in interactions between the limb and other parts of the embryo. There are several processes that necessarily involve such interactions: (a) the migration of muscle progenitor cells into the limb: (b) the migration of neural crest cells (precursors of pigment cells) into the limb; (c) the invasion of axons into the limb; and (d) the invasion of blood vessels into the limb. The molecular basis of these processes has not been investigated as thoroughly as that of the patterning ones. However, the presence of inductive signals from the somites toward the limb bud, and vice versa, has been established in the migration of muscle precursor cells ${ }^{34-36}$. The migration of axons into the limb provides a probable case for the involvement of digit patterning gene activation in processes that occur in the lateral ridge. The Robol gene and members of the Slit gene family appear to be involved in axon guidance in mice, Xenopus and Drosophila. The three Slit genes and Robol are expressed in the mouse in unique and complementary patterns in the front and hind limb bud and in the lateral ridge tissue between the limb buds. In Xenopus, Slitl and Robol interact with each other, but otherwise the regulation is unknown. Yuan et al. 37 infer from the expression pattern of Slit3 (at E10.5 and E11.5) that interactions with the antero-posterior-acting molecules, such as $S h h$, are probable and, from the expression pattern of Slit2 and Slit3, that interactions with Shh and BmP genes are likely. If this were the case, it would provide a link between axonal guidance and digit patterning.

\section{Evidence for negative pleiotropic effects}

The interdependent molecular pathways operating during the phylotypic stage lead to the expectation of many pleiotropic effects associated with changes in the number of digits or other limb bones, both within the limb itself and in other parts of the embryo. Indeed, mutations for changes in the number of digits are associated with many serious abnormalities in mice, chickens and humans ${ }^{22,38-40}$. Polydactyly is a characteristic of many human syndromes, such as Ellis van Crefeld, Bardet-Biedl, trisomy 13 and Down syndromes ${ }^{4,41}$. In $15 \%$ of babies born with an extra toe, other congenital abnormalities are also detected ${ }^{4}$, although in $85 \%$ of the babies with polydactyly, there are no associated anomalies. However, the problem in interpreting these data is that later medical problems are not usually recorded. A review on limb reduction in humans, recording abnormalities at birth with a follow-up a year later, revealed an incidence of limb reduction at birth of $0.06 \%(n=1213913)$, of which $12.9 \%$ had associated abnormalities $^{42}$. Of the children with associated abnormalities, $16 \%(n=348)$ died in the first year, and of the children without associated anomalies, $4 \%(n=311)$ died in the first year. This indicates that very serious abnormalities are missed during birth checks (in the general population mortality in the first year is $-0.8 \%$; Ref. 43 ). No information was given on later problems.

An additional problem is that syndromes commonly have variable expression ${ }^{44-46}$, which interferes with an objective evaluation of anomalies associated with polydactyly mutations. It would be useful if the longevity and fertility of patients were included in genetic studies of families with such abnormalities.

In spite of these problems, there is abundant evidence for the importance of negative pleiotropic effects in birds and mammals. In mice, the occurrence of oligodactyly was 

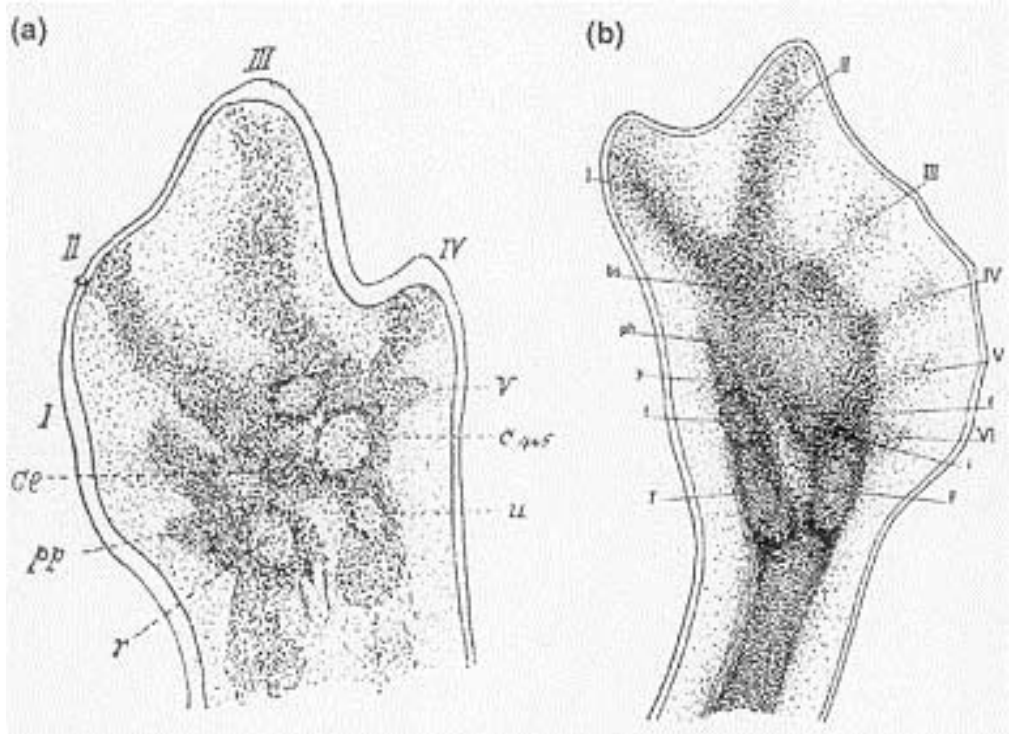

Fig. 5. Embryology of digits in amphibians. (a) Embryonic hand of the anuran Pelobates fuscus, showing still Anlagen of all five digits, and the prepollex as possible sixth digit. The Anlage of the fifth digit will not develop into a finger. Abbreviations: pp, prepollex; r, radius; u, ulna; I-V, digits. Reproduced from Ref. 64. (b) Embryonic foot of the urodele Ambystoma tigrinum, showing Anlagen of six digits and a trace of the prehallux (possibly the $7^{\text {th }}$ digit). Abbreviations: I; fibula; ph, prehallux; 1 ; tibia; t, tibiale; IVI, digits. Reproduced from Ref. 65.

always associated with pleiotropic effects, both in the appendicular and axial skeleton ${ }^{22}$. In chickens, oligo- and polydactyly are also associated with other problems. In these birds, continued selection for polydactyly, for example, led to malformations of the radius $^{22}$. There are the famous polydactylous monsters described by Sewall Wright ${ }^{47}$, who showed how guinea pigs that are heterozygous for the $P x$ mutation are polydactylous without any apparent abnormalities, suggesting that this gene might act only locally on the footplates. However, individuals that are homozygous for the mutations have anomalies in almost every organ in the body. The well-established cases of polydactyly occurring in various dog breeds and the indications for pleiotropic effects are discussed in Box 1.

Overall, therefore, there appears to be sufficient evidence for the negative pleiotropic effects that are expected as a result of molecular interactions during limb development. Further studies are necessary to unravel precisely the relevant interacting molecular pathways.

\section{The exceptional position of amphibians}

In amphibians with aquatic larvae, limb development occurs later than in amniotes and generally occurs after the phylotypic stage. Limb development is especially late in anurans that have an extreme mode of metamorphosis. This suggests that limb development cannot take part in the interactivity of the phylotypic stage, a drastic difference compared with amniotes. This is in agreement with the extremely high selforganizing capacity of the limb buds in many amphibians compared with those in amniotes $^{24}$. Amphibian limb buds can be grafted to very different places, such as the head, and still successfully develop into limbs, which are even capable of movement ${ }^{48}$. 
Limb development can proceed almost as an independent module, with few interactions with other parts of the body. This reduces the possible number of pleiotropic effects of mutations that affect limb development, at least in other parts of the body. However, the pleiotropic effects within the limb are not expected to be lower than those in amniotes. Such effects will probably constrain changes in the number of limb elements; reversals of evolutionary limb reduction are indeed rare and mainly limited to an increase in the number of phalanges (hyperphalangy) ${ }^{49}$. Evolutionary reductions in digit number have occurred frequently in amphibians, but as in aroniotes, these also seem constrained. This follows from the presence of Anlagen of reduced fingers early on during development. For example, the fifth finger that has disappeared in the hand of frogs and salamanders is still visible as Anlage in some frogs and salamanders (Fig. 5a), and the postminimus is still formed as Anlage in some salamanders, just as in humans (Fig. 5b). The mechanism of evolutionary limb reduction, therefore, appears similar to that in aroniotes, as is the loss of limb bones in approximately the reverse order that they are developed, suggesting once again a developmental constraint on limb reduction (Box 3). In spite of the apparent presence of an evolutionary constraint on changes in the number of digits, the absence of inductive interactions with other parts of the amphibian embryo suggests that the constraint will be weaker in amphibians than in aroniotes. Is there support for such an expectation? Variation, including intraspecific variation in the number of phalanges, is more common in amphibians than in aroniotes $\mathrm{s}^{50-54}$. Variation in the number of carpal and tarsal bones is also remarkably high ${ }^{50-55}$. In particular, hyperphalangy occurs frequently, and this represents the only common form of evolutionary reversal of limb reduction. Although hyperphalangy also occurs in aroniotes, it is considerably less common than in amphibians and occurs only in extant species of whales and geckos. The number of phalanges and carpal and tarsal bones is determined very late during limb patterning, when most of the patterning has been completed (including the number of digits). Changes in these distal elements are, therefore, expected to be less constrained than in those elements that are patterned earlier.

\section{Are there polydactylous frogs?}

The presence of a PREHALLUX and PREPOLLEX in frogs could represent cases of polydactyly at a species level. Many frogs have a prepollex (Fig. 6) in their hand and a prehallux in their foot. These are small digit-like structures anterior to the 'first digit'. Based on the available evidence in the literature it seems best to treat the prepollex and prehallux in the same way as the other digits (Box 4).

The presence of a prepollex and prehallux is distributed mosaically over the more advanced frog taxa. There is large inter- and intraspecific variation in the number of phalanges of the prepollex and prehallux, varying from zero in many species to an exceptional five in the leptodactylyd Telmatobius hautholi ${ }^{55}$. A striking example of intraspecific variation is the presence of a prepollex in males and its absence in females, seen in several leptodactyloid frogs ${ }^{56}$ (Fig. 5). This pattern is explained by the function of the prepollex: males use it during mating to clasp the females ${ }^{57}$. The mosaic distribution of the prepollex and the high variability within genera and species suggests that both increases (polydactyly) and decreases in the number of phalanges must have occurred many times. 


\section{Box 4. Do frogs have six toes?}

The confusion about whether the prepollex and prehallux are digits seems to be caused mainly by the once-powerful paradigm of an archetypal tetrapod hand and foot with five digits. Frogs usually have four digits on their hand, in addition to the prepollex. Very often, the prepollex has been hypothesized to be digit I, with a total number of five digits on the hand. The problem arises when one considers the foot, because most frogs have five digits in addition to the prehallux. This has led to the conclusion that the prehallux can thus not be a toe, as this would lead to six toes.. It is now well known that early tetrapods had more than five digits, and that there is no archetypal hand with five digits. Interestingly, embryologists at the end of the 19 th century and beginning of the 20th century concluded, on the basis of developmental data, that the ancestral condition of the tetrapod hand was at least seven digits, including the usual five digits, prepollex/prehallux and the postminimus $^{\mathrm{b}-\mathrm{d}}$. Additional confusion comes from the model of Shubin and Alberch, which assumes that all other digits are formed from the so-called digital ray, whereas the prepollex and prehallux are formed from a different condensation. The presumed difference in development in this model could be a reason to decide against serial homology of the prepollex/prehallux with the digits of the digital ray. Shubin and Alberch do not explicitly decide on the nature of the prepollex and prehallux. In addition, there is evidence from several groups of tetrapods that cannot be explained by the model, especially the digits of the extinct amphibians with up to eight digits. Wagner et al. have concluded that, by contrast to amniotes, the model of Shubin and Alberch. is not helpful in explaining digit development of urodeles. In mammals, a careful developmental series of the foot of the rodent Cavia porcellus and the monkey Microcebus myoxinus clearly shows that not only the prepollex emerges from a different condensation from the other digits, but so does digit one (Ref. g). In addition, explantation and extirpation experiments have suggested that already before the condensation phase of bone development there exists a rather fine-scaled mosaic prepattern with the possibility of the anterior part developing independently of the posterior part and vice versa (e.g. Ref. h). This argues against the validity of the digital arch model. The published data on anurans are not conclusive for or against the mode ${ }^{\mathrm{i}, \mathrm{j}}$. Therefore, the support for the model of the digital arch is insufficient to contradict the digital nature of the prehallux and prepollex.

The fossil data on Ichthyostega and Eryops show that the prepollex and prehallux are very digit-liked ${ }^{\mathrm{d}, \mathrm{k}}$ in these early amphibians. The similarities in shape, position and construction are striking, not on I y in the fossil remains of the earliest amphibians, but also in the early developmental stages. The early developmental stages of the hand of the anurans Pelobates fuscus and Bombina pachypus showthat in the condensation phase the development of the prepollex and prehallux proceeds in the same way as in the other digits (Fig. 6). The remarkable similarity of the prepollex/prehallux and digits in both the fossil and developmental records leads us to consider the prehallux and prepollex as digits, equal to the other digits. 


\section{References}

a Trueb, L. (1973) Bones, frogs and evolution. In Evolutionary Biology of the Anurans (Vial, J.L., ed.), pp. 65-132, University of Missouri Press

b Emery, C. (1890)Zur Morphologiedes Hand- und Fussskeletts. Anat. Anz. 5:283-294

c Steiner, H. (1921) Hand und Fuss der Amphibien, ein Beitrag zur Extremitätenfrage. Anat. Anz. 22:513-542

d Gregory, W.K et al. (1922) The carpus of eryops and the structure of the primitive chiropterygium. Bull. Am. Nat. Hist. 68:279-288

e Shubin, N. and Alberch, P. (1986) Amorphogenetic approach on the origin of and basic organization of the tetrapod limb. Evol. Biol. 20:319-387

f Wagner, G.P. et al. (2000) Developmental evolution as a mechanistic science: The inference from developmental mechanisms to evolutionary processes. Am. Zool. 40:819-831

g Schmidt-Ehrenberg, E.C. (1942) Die Embryogenese des Extremitätenskelettes der Säugetiere. Rev. Suisse Zool. 49:33-132

h Saunders, J. W. (1948) The proximo-dista1 sequence of origin of the parts of the chick wing and the role of the ectoderm. J. Exp. Zool. 108:325-346

i Fabrezi, M. (1993)The anuran tarsus. Alytes 11:47-63

j Jarosova, J. (1974) The components of the tarsus in Paleobatrachus and their development in related recent species. Acta Univ. Carolinae-Geol. 1:119-144

k Jarvik, E. (1980) Basic Structure and Evolution of Vertebrates (Vol. 2), Academic Press

The variation in presence and size of the prepollex and prehallux in frogs, and the high variation in phalanges and carpal and tarsal elements in amphibians in general, suggests that the constraint against changes in the number of more distal elements is weaker in amphibians than in amniotes. Moreover, the differences in hand and foot shapes in amphibians are small compared with those of amniotes (e.g. bat wings and bird feet). In addition, in amphibians, there are no examples of the extra digit- like structures that have evolved many times in amniotes. Thus, although the selective force on changes in limb bones as apparent from changes in limb morphology appears smaller in amphibians than in amniotes, the variability in the number of limb elements is greater. Amniotes have drastically modified hand and foot bones, have a low variability in the number of hand and foot bones, have evolved extra digit-like structures and do not display polydactyly, whereas amphibians have no drastically modified hand and foot bones, have a high variability in the number of hand and foot bones, have no extra digitlike structures and probably show polydactyly. These data support the hypothesis that the decoupling of limb development from the interactivity of the phylotypic stage owing to later limb development has relaxed the negative selection pressure against changes of limb patterning. Such relaxation in selection pressure is all the more remarkable, because it is in striking contrast to the extreme conservation of the overall body plan of amphibians, in particular that of frogs. 


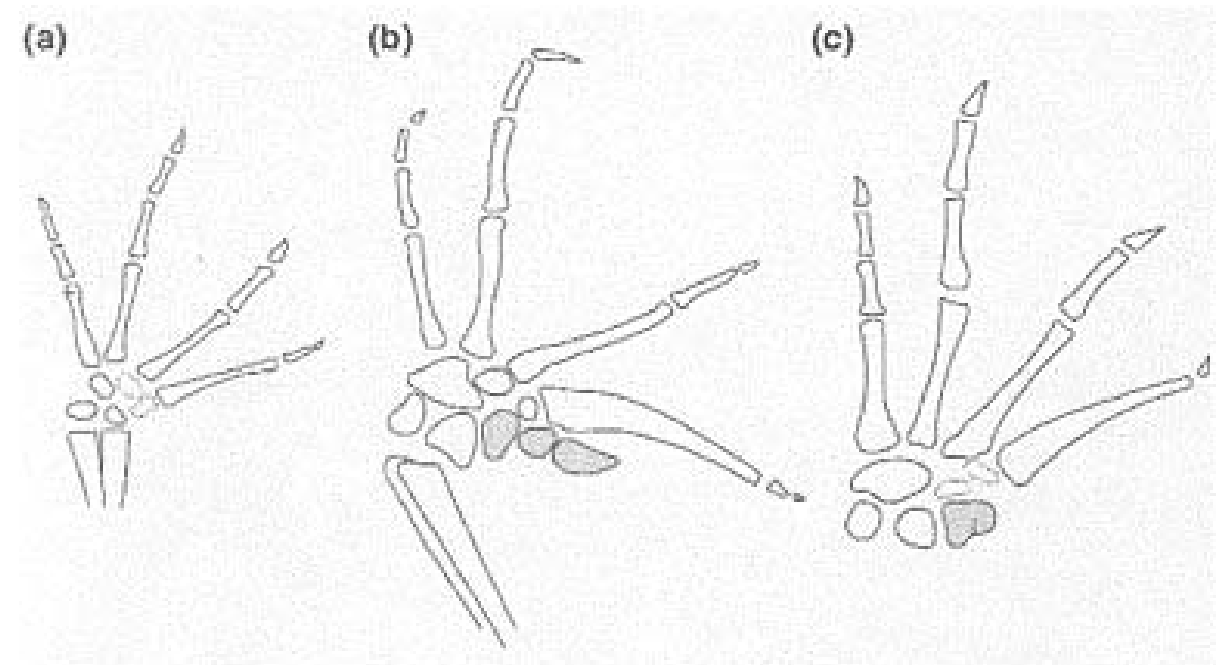

Fig. 6. Intraspecific variation in the prepollex of the leptodactylid frog Limnodynastes tasmaniensis. (a) Females do not have a prepollex. (b) and (c) Males usually have three elements (presumably two phalanges), but can have fewer elements, as shown in (c). The prepollex is indicated in grey. The broken lines are enhanced. Reproduced, with permission, from Ref. 57, by courtesy of The Natural History Museum, London.

\section{Conclusion}

There is new support in the literature for the hypotheses of Lande ${ }^{1}$ and Wright ${ }^{2}$, that polydactyly and other changes of limb patterning are constrained by negative pleiotropic effects. These effects not only explain the absence of polydactyly and other atavisms, but can also explain the extreme conservation of the number of bones more proximal to the hand: the one proximal bone (femur or humerus), followed by two more distal ones (radius and ulna or tibia and fibula). The number of wrist bones and anklebones is more variable, but most of the variability is the result of fusions and not of change $\mathrm{B}$ in the number of bone Anlagen. Changes have occurred during the evolution of tetrapod limbs, most strikingly in amniotes. Changes in size and shape of elements are apparently easy to realize evolutionarily, as are changes in attachment of muscles and ligaments. Genetic changes occur at a high frequency, as do plastic changes in response to changed functional demands. Changes during later stages of development that influence the form and function of structures are, therefore, considerably easier to realize in evolution than are changes during the phylotypic stage.

It appears that the negative pleiotropic effects causing the evolutionary constraint in amniotes and amphibians primarily result from the many inductive interactions occurring during limb development. In amniotes, limb development is integrated into the inductive interactivity of embryonic development as a whole, and these interactions increase the number of negative pleiotropic effects associated with changes of limb patterning. There are many interactions occurring among modules, and therefore modularity is limited. In amphibians, the limb develops as a semi-independent module, and therefore pleiotropic effects of limb changes will mainly be limited to the limb itself. We conjecture, therefore, that the constraint in amniotes is stronger than that in amphibians. The higher variability in the number of phalanges, anklebones and wrist bones in amphibians supports this conjecture. The probable cases of polydactyly in frogs and the absence of extra digit-like structures in amphibians provide further support for the hypothesis. 


\section{Medical and veterinary relevance}

We suggest that these findings are relevant to human and veterinary medicine. The presence of extra toes is generally seen as a rather innocuous variation (as is the presence of cervical ribs, see below). However, the general presence of negative pleiotropic effects suggests the possibility of long-term effects. For humans, the diagnosis of extra digits should alert doctors to the possibility of medical problems in later life. In addition, prenatal checks for anomalies appear prudent. In dogs and other domestic animals, selecting for a normal number of toes might help eradicate anomalies occurring later in life.

\section{Conservation of body plans}

The strong evolutionary conservation of the number of digits and limb bones in amniotes provides an example of the conservation of a character determined during the phylotypic stage. Therefore, not only is the stage itself conserved, but also certain characters that are determined during that stage. A case has already been made for the seven cervical vertebrae in mammals ${ }^{16,58}$. The conservation of the number of limb bones in amniotes can be added as an example. The crucial importance of global inductive interactions for the development of the entire organism during the phylotypic stage can be further deduced from the pattern of reduction of other structures of which the Anlage appears during this stage. As in limb reduction, the eye and PRONEPHROS are evolutionarily reduced via the laborious process of construction followed by destruction $^{59,60}$, suggesting that we have two eyes for the same reason that we have five digits: that is, the chain of inductive interactions cannot be changed easily. 


\section{Glossary}

Anlage: primordium, the earliest state in the development of structure.

Atavism: evolutionary reversal to the ancestral state.

Inductive interactivity: the presence of mutual or network-like inductive signaling.

Inductive signaling: the process in which one or more cells induce a response in one or more other cells.

Modularity: absence of interactions between modules.

Module: unit of development characterized by a relative absence of inductive interactions toward the module from the outside and a much larger interactivity within the module.

Pleiotropy: the production of several effects by one gene.

Polydactyly: increase in digit number.

Prehallux: digit, or digit-like structure, anterior to the hailux (big toe).

Prepollex: digit, or digit-like structure, anterior to the pollex (thumb).

Pronephros: kidney-like structure functioning as a kidney in fish and amphibian larvae. In mammals and birds, it appears as a transitory structure during embryology before other kidney-like structures (mesonephros and metanephros) and is not thought to be active as a kidney.

Supernumerary phalanges: increased number of phalanges. 


\section{References}

1. Lande, R. (1978) Evolutionary mechanisms of limb loss in tetrapods. Evolution 32: 73-92

2. Wright, S. (1968) Evolution and the Genetics of Populations (Vol. 1), Genetic and Biometric Foundation, University of Chicago Press

3. Castilla, E.E. et al. (1996) Epidemiological analysis of rare polydactylies. Am. J. Med. Genet. 65:295-303

4. Castilla, E.E. et al. (1998) Associated anomalies in individuals with polydactyly. Am. J. Med. Genet. 80:459-465

5. Bateson, W. (1894) Materials for the Study of Variation, Macmillan

6. Schmidt-Ehrenberg, E.C. (1942) Die Embryogenese des Extremitätenskelettes der Säugetiere. Rev. Suisse Zool. 49:33-132

7. Raynaud, A. and Brabet, J. (1994) New data on embryonic development of the limbs in the slow- worm, Anguis fragilis (Linne 1758). Ann. Sci. Nat. Zool. Biol. Anim. 15:97-113

8. Rahmani, T.M-Z. (1974) Morphogenesis of the rudimentary hind-limb of the Glass Snake (Ophisaurus apodus Pallas). J. Embryol. Exp. Morphol. 32:431-443

9. Liem, KF. et al. (200 1) Functional Anatomy of the Vertebrates: an Evolutionary Perspective (3rd edn), Harcourt College Publishers

10. Carroll, R.L. (1997) Patterns and Processes of Vertebrate Evolution, Cambridge University Press

11. Motani, R. (1998) First complete forefin of the ichthyosaur Grippia longirostris from the Triassic of Spitsbergen. Palaeontology 41:591-599

12. May, O. (1996) The pisiform bone: sesamoid or carpal bone? Ann. Chir. Main. Memb. Super. 15,265-271

13. Hall, B.K. (1996) Baupläne, phylotypic stages, and constraint - Why there are so few types of animals. Evol. Biol. 29:215-261

14. Raff, R.A. (1996) The Shape of Life, University of Chicago Press

15. Sander, K. (1983) The evolution of patterning mechanisms: gleanings from insect embryogenesis and spermatogenesis. In Development and Evolution (Goodwin, B.C. et al., eds), pp. 137-154, Cambridge University Press

16. Galis, F. and Metz, J.A.J. (2001) Testing the vulnerability of the phylotypic stage: On modularity and evolutionary conservation. J. Exp. Zool. 291:195-204

17. Wagner, G.P. (1996) Homologues, natural kinds and the evolution of modularity. Am. Zool. 36:36-43

18. Galis, F. (1996) The application of functional morphology to evolutionary studies. Trends Ecol. Evol. 11:124-129

19. Bolker, J.A. (2000) Modularity in development and why it matters to Evo-Devo. Am. Zool. 40:770-776 
20. Ngo-Muller, V. and Muneoka, K (2000) Influence of FGF4 on digit morphogenesis during limb development in the mouse. Dev. Biol. 219:224-236

21. Hall, B.K. and Myake, T. (2000) All for one and one for all: condensations and the initiation of skeletal development. BioEssays 22:138-147

22. Grueneberg, H. (1963) The Pathology of Development: a Study of Inherited Skeletal Disorders in Animals, Blackwell Scientific

23. Keibel, F. (1897-1937) Normentafeln zur Entwicklungsgeschichte der Wirbeltiere, Heft I-XV, Gustav Fisher

24. Balinsky, B.I. (1970) An Introduction to Embryology (3rd edn), W.B. Saunders

25. Agnish, N.D. and Kochhar, D.M. (1977) The role of somites in the growth and early development of mouse limb buds. Dev. Biol. 56:174-183

26. Strangeways, T.S.P. and Fell, H.B. (1926) Experimental studies on the differentiation of embryonic tissues growing in vivo and in vitro. I. The development of the undifferentiated limb-bud (a) when subcutaneously grafted in the post-embryonic chick and (b) when cultivated in vitro. Proc. R. Soc. London B Biol. Sci. 99,340-366

27. Johnson, R.L. and Tabin, C.J. (1997) Molecular models for vertebrate limb development. Cell 90:979-990

28. Cohn, M.J. and Bright, P.E. (1999) Molecular control of vertebrate limb development, evolution and congenital malformations. Cell Tissue Res. 296:3-17

29. Charite, J. et al. (2000) The bHLH transcription factor dHAND controls Sonic hedgehog expression and establishment of the zone of polarizing activity during limb development. Development 127:2461-2470

30. Kraus, P. et al. (2001) Some distal limb structures develop in mice lacking Sonic hedgehog signaling. Mech. Dev. 100:45-58

31. Yokoyama, H. et al. (1998) Multiple digit formation in Xenopus limb bud recombinants. Dev. Biol. 196:1-10

32. Drossopoulou, G. et al. (2000) A model for anteroposterior patterning of the vertebrate limb based on sequential long- and short-range Shh signalling and Bmp signalling. Development 127:1337-1348

33. Amthor, H. et al. (1998) The importance of timing differentiation during limb muscle development. Cun: Biol. 8:642-652

34. Hayashi, K and Ozawa, E. (1995) Myogenic cell migration from somites is induced by tissue contact with medial region of the presumptive limb mesoderm in chick embryos. Development 121:661-669

35. Mennerich, D. et al. (1998) Pax-3 is necessary but not sufficient for Ibx2 expression in myogenic precursor cells of the limb. Mech. Dev. 73:147-158

36. Dietrich, S. et al. (1999) The role of SF/HGF and c-Met in the development of skeletal muscle. Development 126:1621-1629 
37. Yuan, W. et al. (1995) The mouse SLIT family: Secreted ligands for ROBO expressed in patterns that suggest a role in morphogenesis and axon guidance. Dev. Biol. 212:290-306

38. Watanabe, H. et al. (1992) Polydactyly of the foot - An analysis of 265 cases and a morphological classification. Plast. Reconstr: Surg. 89:856-877

39. Lewis, K.E. et al. (1999) Expression of ptc and gli genes in talpid3 suggests bifurcation in Shh pathway. Development 126:2397-2407

40. Muranjan, M.N. and Bharucha, BA. (2000) Unusual hand malformations with cardiac defects - a variant of heart-hand syndrome IV. Indian Pediatr. 67:392-394

41. Rosano, A. et al. (2000) Limb defects associated with major congenital anomalies: clinical and epidemiological study from the international clearing house for birth defects monitoring systems. Am. J. Med. Genet. 93:11-116

42. Froster-Iskenius. U.G. and Baird, P.A. (1989). Limb reduction defects in over one million consecutive live births. Teratology 39:127-135

43. MacDorman, M.F. and Atkinson, J.O. (1999) Infant mortality statistics from the 1997 period linked birth/infant death data set. Natl. Vital Stat. Rep. 47:1-23

44. Digilio, M.C. et al. (1997) Atrioventricular canal defect and postaxial polydactyly indicating phenotypic overlap of Ellis-van Creveld and Kaufman-McKusick Syndromes. Pediatr. Cardiol. 18:74-75

45. Dippie, K.M. and McCabe, E.R.B. (2000) Phenotypes of patients with simple Mendelian disorders are complex traits: thresholds, modifiers, and systems dynamics. Am. J. Hum. Genet. 66:1729-1735

46. Kaplan, B.S. and Bellah. R.D. (1999) Postaxial polydactyly, ulnar ray dysgenesis, and renal cystic dysplasia in sibs. Am. J. Med. Genet. 87:426-239

47. Wright, S. (1935)A mutation of the guinea pig, tending to restore the pentadactyl foot when heterozygous producing a monstrosity when homozygous. Genetics 20:84-107

48. Detwiler, S.R. (1930) Observations upon the growth function and nerve supply of limbs when grafted to the head of salamander embryos. J. Exp. Zool. 55:319-370

49. Trueb, L. (1973) Bones, frogs and evolution. In Evolutionary Biology of the Anurons (Vial, J.L., ed.), pp. 65-132, University Missouri Press

50. Gollmann, G. (1991) Osteological variation in Geocrinio laevis, Geocrinia victoriona, and their hybrid populations (Amphibia, Anura, Myobatrachinae) $\mathrm{Z}$. zool. Syst. Evolutionsforsch. 29:289-303

51. Rienesl, R. and Wagner, G.P. (1992) Constancy and change of basipodial variation patterns: a comparative study of crested and marbled newts - Triturus cristatus, Triturus mannorotus - and their natural hybrids. J. Evol. Biol. 5:307-324

52. Shubin, N. et al. (1995) Morphological variation in the limbs of Taricha granulosa (Caudata: Salamandridae): evolutionary and phylogenetic implications. Evolution 49:874-884 
53. Hanken, J. (1982) Appendicular skeletal morphology in minute salamanders, genus Thorius (Amphibia: Plethodontidae): growth regulation, adult size determination and natural variation. J. Morphol. 174:57-77

54. Hanken, J. (1983) High incidence of limb skeletal variants in a peripheral population ofthe red-backed salamander, Plethodon cinereus (Amphibia: Plethodontidae), from Nova Scotia. Can. J. Zool. 61:1925-1931

55. Lynch, J.D. (1971) Evolutionary relationships, osteology, and zoogeography of leptodactyloid frogs. Univ. Kansas Mus. Nat. Hist. Misc. Publ. 53:1-238

56. Alberch, P. and Gale, E.A. (1983) Size dependence during the development of the amphibian foot. Cochicine-induced digital loss and reduction. J. Embryol. Exp. Morphol. 76:177-197

57. Parker, H.W. (1940) The australasian frogs of the family Leptodactylidae. Nouitates Zoologicae 42:1-107

58. Galis, F. (1999) Why do almost all mammals have seven cervical vertebrae? Developmental constraints, Hox genes and cancer. J. Exp. Zool. 285:19-26

59. Langecker, T.G. etal. (1993) Transcription of the opsin gene in degenerate eyes of cave-dwelling Astyanax fasciatus (Teleostei, Characidae) and of its conspecific epigean ancestor during early ontogeny. Cell Tissue Res. 273:183-192

60. Carroll. T. et al. (1999) Molecular regulation of pronephric development. Cun. Top. Deu. Bial. 44:67-100

61. Seiferle, E. (1927) Atavismus und Polydaktylie der hyperdaktilen Hinterpfote des Haushundes. Morph. Jb. 57:313-380

62. Flower, W.H. (1888) Einleitung in die Osteologie der Säugetiere. Wilhe1m Engelmann

63. Owen, R. (1866) On the Anatomy of Vertebrates, Longmans Green

64. Emery, C. (1890) Zur Morphologie des Hand- und Fussskeletts. Anat. Anz. 5:283294

65. Steiner, H. (1921) Hand und Fuss der Amphibien, ein Beitrag zur Extremitätenfrage. Anat. Anz. 22:513-542 